\title{
Monte Carlo simulation of inelastic collisions of electrons in solids
}

\author{
F. Salvat*, D. Bote*, and J.M. Fernández-Varea* \\ * Facultat de Física (ECM), Universitat de Barcelona. Diagonal 647. 08028 Barcelona. Spain.
}

Inelastic interactions are the dominant energy-loss mechanism for kilovolt electrons in solids, and the cause of primary characteristic x-ray emission in EPMA. In spite of their importance, Monte Carlo codes for the simulation of EPMA spectra usually implement oversimplified models to describe the effect of inelastic collisions, the simplest of which is the continuous-slowing-down approximation. Although interactions with conduction electrons and weakly bound shells are sensitive to the structure of the solid, the ionization of inner shells can be accurately described by considering the target atom as isolated, i.e., using conventional atomic collision theory.

Most of the atomic collision models currently in use are based on the plane-wave Born approximation (PWBA), because of its conceptual simplicity and because calculations for free atoms are relatively simple $[1,2]$. The main limitation of the PWBA is that it neglects the effect of the atomic field on the wave functions of the projectile. This effect is accounted for in a consistent manner by the distorted-wave Born approximation (DWBA); unfortunately, calculations based on this approximation are feasible only for electrons with kinetic energies up to about 20 times the ionization energy of the active target electron [3]. In recent years, we have performed systematic calculations of cross sections for inner shell ionization by electron impact using a combination of the DWBA and the PWBA for K, L and M shells of neutral atoms. We have also calculated a complete database of generalized oscillator strengths for all shells of neutral atoms, from which we can easily obtain differential and integrated cross sections for ionization of inner shells [4].

Excitations of weakly-bound electrons can be described by using optical-data models, which combine experimental or empirical dielectric functions $\epsilon(\omega)$ with suitable extension algorithms [5] to set a realistic model for the generalized oscillator strength. This scheme has the advantage of incorporating specificities of the solid that are difficult to calculate from first principles, and the drawback of requiring experimental information which is only available for a limited set of materials. The extension algorithms used to date are based on the Lindhard dielectric function, which is derived from first-order perturbation theory. By combining an optical-data model for excitations of outer electrons with the PWBA for inner shell ionization, we obtain a consistent description of inelastic collisions of electrons in the solid. The range of validity of the composite model is the same as for the PWBA, that is, results are reliable whenever the kinetic energy $E$ of the projectile is much larger than the ionization energy of the active target electron (the Fermi energy in the case of conduction electrons).

On the basis of such a composite model, with the aid of Bethe's sum rule, we can readily derive asymptotic formulas (i.e., valid for high-energy projectiles) for the inelastic mean free path and the stopping power [5]. Each of these formulas contains two parameters, that are characteristic of the considered material. These formulas are obtained by neglecting terms of 
orders $E^{-1}$ and higher and, consequently, at low energies their values deviate from the mean free paths and stopping powers calculated by integrating the differential cross sections. These deviations arise mostly from contributions from inner shells. Since the response of these shells is fairly insensitive to the state of aggregation, "shell corrections" can be readily obtained from the calculated PWBA atomic cross sections.

Simple strategies for the simulation of electron inelastic scattering, based on the derived asymptotic formulas or using existing databases, will be described. Particularly fast, and still accurate, simulations can be obtained with class II schemes [6], which combine detailed simulation of hard interactions (with energy losses above a certain cutoff) with condensed simulation of soft interactions. A prerequisite for the desired simplicity is that the ionization of inner shells (and the subsequent generation of primary characteristic x rays) has to be considered as a separate process. This implies that correlations between x-ray emission and energy deposition by the transported electron will be disregarded.

\section{References}

[1] U. Fano, Ann. Rev. Nucl. Sci. 13 (1963) 1.

[2] D. Bote et al. Phys. Rev. A 77 (2008) 042701.

[3] S. Segui et al, Phys. Rev. A 67 (2003) 062710.

[4] D. Bote et al., Internal report, Universitat de Barcelona.

[5] J.M. Fernández-Varea et al., Nucl. Instrum. Methods Phys. Res. Sect. B 229 (2005) 187.

[6] F. Salvat et al., PENELOPE - A Code System for Monte Carlo Simulation of Electron and Photon Transport, OECD/Nuclear Energy Agency, Issy-les-Moulineaux, 2008.

[7] This work has been partially supported by the Spanish Ministerio de Educación y Ciencia (FPA2006-12066) and FEDER, and the AGAUR, Generalitat de Catalunya, (SGR2005-00013). 